TITLE:

\title{
Concatenated solutions of delay- differential equations and their representation with time-delay feedback systems
}

\section{$\operatorname{AUTHOR}(S):$}

Hagiwara, Tomomichi; Kobayashi, Masahiro

\section{CITATION:}

Hagiwara, Tomomichi ...[et al]. Concatenated solutions of delay-differential equations and their representation with time-delay feedback systems. International Journal of Control 2011, 84(6): 1126-1139

ISSUE DATE:

2011-06

URL:

http://hdl.handle.net/2433/158043

\section{RIGHT:}

(C) 2011 Taylor \& Francis Ltd; This is not the published version. Please cite only the published version.; この論文は出版社版でありません。引 用の際には出版社版をご確認ご利用ください。 


\title{
RESEARCH ARTICLE
}

\section{Concatenated Solutions of Delay-Differential Equations and Their Representation with Time-Delay Feedback Systems}

\author{
Tomomichi Hagiwara ${ }^{\dagger}$ and Masahiro Kobayashi \\ (Received 00 Month 200x; final version received 00 Month 200x)
}

\begin{abstract}
Stimulated by the monodromy operator approach to time-delay systems (TDSs) developed recently, this paper studies the conversion problems of delay-differential equations (DDEs) into the representation as time-delay feedback systems. We give two conversion methods including the conversion of initial conditions, where we show that each of the two methods corresponds, in general, to one of two different definitions for the solutions of DDEs, called pseudo concatenated solutions and continuous concatenated solution. The study is actually closely related to the subtle behaviors of the solutions of DDEs under discontinuous initial conditions, and simple examples illustrating such subtleties as well as the validity of the conversion methods are also provided. The results of this paper suggest that the ability of representing TDSs is higher in the representation as time-delay feedback systems than in the representation as DDEs.
\end{abstract}

Keywords: time-delay systems, delay-differential equations, discontinuous initial functions, pseudo concatenated solutions, continuous concatenated solutions.

\section{Introduction}

Time-delay systems (TDSs) are very commonly encountered in engineering and sciences, and hence there is a very long and deep history of studies on TDSs. Among them is the study of delay-differential equations (DDEs) (Bellman and Cooke 1963, Hale 1977, Hale and Lunel 1993), which are described by

$$
\dot{x}(t)=J x(t)+\sum_{i=1}^{\eta} K_{i} \dot{x}(t-i h)+\sum_{i=1}^{\eta} L_{i} x(t-i h)
$$

if we confine ourselves to linear time-invariant (LTI) TDSs with commensurate delays.

Aside from many other conventional approaches to TDSs, a new viewpoint for dealing with TDSs was initiated in Hirata and Kokame (2003), in which continuous-time TDSs are treated (through the lifting technique (Yamamoto 1994, Bamieh and Pearson 1992) developed for sampled-data systems) as a sort of discrete-time systems by considering the transitions of their infinite-dimensional state at every $h$ (i.e., the base delay length). This viewpoint has been further extended, and a new bounded-operator-theoretic approach that we call the monodromy operator approach has been developed in Hagiwara (2008), Hagiwara and Hirata (2011), Hagiwara and Fujinami (2010), Hagiwara (2010), Hagiwara and Inui (2010) by applying various recent advances in the study of sampled-data systems. Such an approach is quite promising, e.g., in developing a design method for finite-dimensional discrete-time controllers for continuous-time TDSs. However, the underlying assumption of the monodromy operator approach is that a TDS is represented as a time-delay feedback system $\Sigma$ as in Fig. 1, rather than DDEs. Here, $F$ is a finite-dimensional LTI system, while $H$ is a delay with delay length $h$. Hence, it is very im-

Corresponding author. E-mail: hagiwara@kuee.kyoto-u.ac.jp, Phone: +81-75-383-2250.

Department of Electrical Engineering, Kyoto University, Kyotodaigaku-Katsura, Nishikyo-ku, Kyoto 615-8510, Japan. 
portant to study how the DDE (1) could be converted into the representation as a time-delay feedback system $\Sigma$ because one can then apply the recently developed various techniques in the monodromy operator approach also to TDSs represented by DDEs.

Although the DDE (1) might be believed to be representable as the TDS $\Sigma$ and vice versa, explicit and thorough studies on dealing with such conversion topics are, to the best knowledge of the authors, uncommonly encountered and surprisingly scarce in the literature, in spite of the simplicity of (1). As it turns out in the discussions of this paper, such circumstances seem to be pertinent to the lack of the studies on some subtleties about the solutions of (1). Namely, the DDE (1) could have different types of solutions, depending on some sort of irregularity about the initial state, but the definitions for such different types of solutions are not necessarily explicated in the literature. This is true particularly for the case with discontinuous initial functions. However, even simple and explicit examples are not necessarily provided in the literature that can demonstrate the subtle situation in the relationship among the definitions of the solutions, the irregularity (or discontinuity) of the solutions and that of the initial states. Rare exceptions include the studies in Kolmanovskii and Myshkis (1999), Baker and Paul (2006) and Krishna and Anokhin (1994), but none of them studies satisfactorily the different types of the solutions of (1), nor their relevance to the relationship between (1) and the system $\Sigma$.

Hence, aside from the motivation of giving a solid basis for the monodromy operator approach also to the DDE (1), it is important on its own to clarify the relationship between the treatment of TDSs with the DDE (1) and that with the representation $\Sigma$. Such a study also turns out to involve the studies on different types of the solutions of (1) raised above. We in fact introduce the definitions for the solutions of the DDE (1) called pseudo concatenated solutions and continuous concatenated solutions. The relevance of such definitions to the problem of converting the DDE (1) into the representation as the time-delay feedback system $\Sigma$ (including the discussions about how to convert the not necessarily continuous or everywhere differentiable initial state of (1) into the initial condition of $\Sigma$ ) can be expressed as follows. That is, we consider two types of representations of (1) as $\Sigma$ (denoted by $\Sigma^{\mathrm{I}}$ and $\Sigma^{\mathrm{II}}$ ), and establish that each representation corresponds to a different definition for the solution of (1), in general.

In the view of the present authors, it seems to be a good idea and of pedagogical value to deal with the above conversion problems and the existence/uniqueness problems of pseudo and continuous concatenated solutions of (1) in a parallel fashion. In fact, such treatment provides the paper with a unique feature in the way it conducts the discussions, and leads us to a concise collection of the properties of LTI TDSs from different viewpoints, with the associated supporting examples. In other words, this paper, confining to the case of LTI TDSs with commensurate delays, successfully gives an almost entire picture about the theoretical subtleties on the definitions and properties of the solutions of the DDE (1). The arguments cover both the neutral case and the retarded case, and can be used for easy reference and pedagogical purposes. The arguments are based on nothing but the well-known method of steps, and are more or less relevant to the development in, e.g., Bellen and Zennaro (2003) (carried out rather from a numerical point of view), but the overall theoretical discussions are believed to be original. The derived results might be useful also as giving simple numerical computation methods for (different types of) solutions of (1).

In the arguments of this paper, we encounter many subtle points about differentiability and continuity of functions as well as the associated notational conventions. We believe that the rigorousness of the arguments would definitely be sacrificed if all such points were simply left entirely untouched. On the other hand, however, we also believe that if we give proper additional

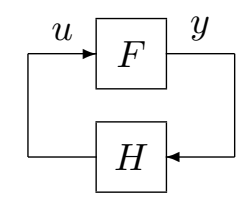

Figure 1. Time-delay feedback system $\Sigma$. 
explanations for enhancing rigorousness whenever such subtle points could possibly lead to ambiguities, then it would be overly distracting. Our decision to reconcile these two aspects and have a moderate balance is to give the additional explanations about the subtleties as footnotes, even though the decision will make this paper to have more than modest numbers of footnotes.

\section{Conversion Problem of DDEs into Time-Delay Feedback Systems}

Let us consider the neutral delay-differential equation (DDE) given by (1), where $\eta \in \mathbb{N}, x(t) \in$ $\mathbb{R}^{n}, J \in \mathbb{R}^{n \times n}, K_{i}, L_{i} \in \mathbb{R}^{n \times n}(i=1, \cdots, \eta)$. We study the Cauchy problem (initial value problem) for (1) under the initial condition given by

$$
\begin{aligned}
& x(t)=\phi(t),-\eta h \leq t<0 \\
& x(0)=\xi
\end{aligned}
$$

where the continuous function $\phi(t)$ is defined on the closed interval $-\eta h \leq t \leq 0$ and continuously differentiable $^{1}$ on this interval. However, we do not, in general, assume that $\xi=\phi(0)$; this implies that the initial function $x(t),-h \leq t \leq 0$ is not necessarily left-continuous at $t=0$, and $\phi(t)$ is not exactly identical with the initial function (for a possible discrepancy at $t=0$ ). Alternatively, we also consider the case with a slightly weaker assumption on $\phi(t)$, in which it is assumed to be bounded and continuously differentiable on each of the intervals $[-i h,-(i-1) h), i=1, \cdots, \eta$. In addition, we also deal with the Cauchy problem for the case of retarded DDE (i.e., the case with $\left.K_{i}=0, i=1, \cdots, \eta\right)$ under a weaker condition on $\phi(t)$.

This paper is interested in representing the (suitably defined) solution $x(t)$ of (1) by means of the feedback system $\Sigma$ shown in Fig. 1, where $F$ is the finite-dimensional linear time-invariant (FDLTI) system given by

$$
\begin{aligned}
& \dot{q}(t)=A q(t)+B u(t) \\
& y(t)=C q(t)+D u(t)
\end{aligned}
$$

and $H$ is the pure delay with retardation $h$ (i.e., $u(t)=y(t-h)$ ). This problem is important not only theoretically but also from the practical purpose of establishing a way to apply the monodromy operator approach developed for the time-delay feedback system $\Sigma$ also to DDEs (as mentioned in the introduction).

Note that we have considered only the discontinuity of the initial function $x(t),-\eta h \leq t \leq 0$ either at $t=0$ (i.e., $\xi \neq \phi(0))$ or $t=-i h(i=1, \cdots, \eta-1)$. This is partly because we do not wish to complicate the problem too much (and thus we decide to assume to some extent the continuity of the initial function) but more essentially because considering some sort of discontinuity particularly at those time instants seems quite important in studying the relationship between the DDE (1) and the time-delay feedback system $\Sigma$. The reason for the latter can be explained by the fact that when $\Sigma$ with some initial conditions is converted into the DDE (1), the corresponding initial function quite often has such discontinuity; taking account of this fact, it is understood to be natural to deal with such discontinuity also in the opposite conversion problem of the DDE (1) into $\Sigma$.

\section{Definitions of the Solutions of DDEs}

As mentioned in the preceding section, this paper considers discontinuous initial functions, in general. To the surprise of the present authors, and to the best of their knowledge, definitions

\footnotetext{
${ }^{1}$ At $t=-\eta h$, continuous differentiability is defined as the existence of right-derivative and its coincidence with $\lim _{t \rightarrow-\eta h+0} \dot{\phi}(t)$; however, it is easy to show that the existence of the above limit in fact ensures the existence of the right-derivative at $t=-\eta h$ and its coincidence with this limit. Likewise, continuous differentiability at $t=0$ refers to the existence of $\lim _{t \rightarrow-0} \dot{\phi}(t)$.
} 
for the solutions of (1) for such a general case, with fully unambiguous notions and statement, are scarcely encountered in the literature. Before proceeding to the conversion problem of DDEs into time-delay feedback systems, this section is devoted to the definitions of a few types of solutions of the DDE (1), depending on the different assumptions on the initial functions.

\subsection{Neutral DDE}

We first give a few definitions on the solutions of the neutral DDE (1).

Definition $1 x(t), t \geq-\eta h$ is said to be a regular solution of (1) if the following three conditions are satisfied: (i) it satisfies the initial conditions (2) and (3) for $\xi(=\phi(0))$ and $\phi(t)$ that is continuously differentiable on the interval $[-\eta h, 0]$; (ii) it is differentiable $e^{1}$ for $t \geq-\eta h$; (iii) it satisfies(1) for $t \geq 0$.

Remark 1 A regular solution is continuous at $t=0$ by definition. Hence, it exists only if $\xi=\phi(0)$.

Next, let us formally rewrite (1) into

$$
\frac{d}{d t} v(t)=J x(t)+\sum_{i=1}^{\eta} L_{i} x(t-i h), \quad v(t)=x(t)-\sum_{i=1}^{\eta} K_{i} x(t-i h)
$$

On the basis of this form, we introduce the following different (weaker in a sense) definition for the solution of (1).

Definition $2 x(t), t \geq-\eta h$ is said to be a concatenated solution of (6), or a pseudo concatenated solution of (1) if the following three conditions are satisfied: (i) it satisfies the initial conditions (2) and (3) for $\xi$ and $\phi(t)$ that is bounded and continuously differentiable on each of the intervals $[-i h,-(i-1) h)(i=1, \cdots, \eta) ;(i i) v(t)=x(t)-\sum_{i=1}^{\eta} K_{i} x(t-i h)$ is continuous for $t \geq 0$; (iii) $v(t)$ is differentiable and satisfies the first equation of (6) for $t \geq 0$ except possibly for $t=k h(k \in \mathbb{N})$.

Remark 2 For $t=0$, the condition (iii) should be interpreted to mean that the right-derivative of $v(t)$ exists there and coincides with the right hand side of the first equation in (6) evaluated at $t=0$. Similarly for the following Definitions 3, 4 and 5 (with $v(t)$ replaced by $x(t)$ whenever $x(t)$ is referred to).

Remark 3 It is easy to see in the above definition that $v(t)$ is differentiable for $t \geq 0$ except possibly for $t=k h$ if and only if $x(t)$ is. The continuity condition of $v(t)$, however, is not equivalent to that of $x(t)$, and hence the pseudo concatenated solution of (1) is not necessarily continuous. This definition corresponds to the natural solution defined in Baker and Paul (2006) for more general neutral DDEs with only a single delay, but that reference does not give an alternative definition corresponding to Definition 3 given below.

We also define the (continuous) concatenated solution of (1) (without reference to the modified form (6)) as follows.

Definition $3 x(t), t \geq-\eta h$ is said to be a (continuous) concatenated solution of (1) if the following three conditions are satisfied: (i) it satisfies the initial conditions (2) and (3) for $\xi$ and $\phi(t)$ that is bounded and continuously differentiable on each of the intervals $[-i h,-(i-1) h)(i=$ $1, \cdots, \eta)$ and has limits ${ }^{2} \lim _{t \rightarrow-(i-1) h-0} \phi(t)(i=1, \cdots, \eta)$; (ii) it is continuous for $t \geq 0$; (iii)

\footnotetext{
${ }^{1}$ For $t=-\eta h$, the differentiability means the existence of the right derivative of $x(t)$ there.

${ }^{2}$ Unlike in Definition 2, the existence of these limits play an important role to make the definition meaningful (i.e., the existence of a solution cannot be ensured otherwise). See Section 5.2. We also remark that this requirement is weaker than the boundedness requirement of $\dot{\phi}(t)$ on each interval $[-i h,-(i-1) h)$.
} 
it is differentiable and satisfies (1) for $t \geq 0$ except possibly for $t=k h(k \in \mathbb{N})$.

Remark 4 Once we fix the initial conditions (2) and (3) in a way consistent with each of the above definitions, a regular solution, a pseudo concatenated solution or a continuous concatenated solution exists accordingly, which is in fact unique. The existence is ensured by the following Theorems 1, 2 and 6, respectively, while the uniqueness is a direct consequence from the linearity of (1); more precisely, since the solution (regardless of regular, pseudo concatenated or continuous concatenated solution) for $\phi(t)=0$ and $\xi=0$ is only $x(t)=0$, the superposition principle of the solutions leads to the uniqueness immediately. Furthermore, it is obvious that if a regular solution exists under some initial condition, then it is at the same time a pseudo concatenated solution and a continuous concatenated solution for the same initial condition. However, even if a continuous concatenated solution exists under some initial condition, it is not necessarily a pseudo concatenated solution at the same time; see the relationships between the following Examples 2 and 8, as well as Examples 3 and 9, and, more explicitly, Proposition 1. Hence, Definitions 2 and 3 are generalizations of Definition 1 that are independent of each other, in general.

\section{$3.2 \quad$ Retarded DDE}

We next consider the particular case of $K_{i}=0, i=1, \cdots, \eta$ in (1), i.e., the retarded DDE given by

$$
\dot{x}(t)=J x(t)+\sum_{i=1}^{\eta} L_{i} x(t-i h)
$$

As far as we consider the above equation for $t \geq 0$, we need not assume the differentiability of $x(t)$ for $t<0$, unlike the case of the neutral DDE (1). Hence, we define the regular solutions of the retarded DDE (7) as follows.

Definition $4 x(t), t \geq-\eta h$ is said to be a regular solution of (7) if the following three conditions are satisfied: (i) it satisfies the initial conditions (2) and (3) for $\xi(=\phi(0))$ and $\phi(t)$ that is continuous on the interval $[-\eta h, 0]$; (ii) it is differentiable for $t \geq 0$; (iii) it satisfies (7) for $t \geq 0$.

The differentiability assumption of $x(t)$ for $t<0$ is not necessary also in defining the concatenated solutions of the retarded DDE (7). We also note in Definition 2 that $v(t)$ is continuous if and only if $x(t)$ is, as far as the retarded case $K_{i}=0, i=1, \cdots, \eta$ is concerned. Hence, (Definitions 2 and 3 degenerate to an almost identical one and thus) we introduce the following definition.

Definition $5 x(t), t \geq-\eta h$ is said to be a (continuous) concatenated solution of (7) if the following three conditions are satisfied: (i) it satisfies the initial conditions (2) and (3) for $\xi$ and $\phi(t)$ that is bounded and continuous on each of the intervals $[-i h,-(i-1) h)(i=1, \cdots, \eta)$; (ii) $x(t)$ is continuous for $t \geq 0$; (iii) $x(t)$ is differentiable and satisfies (7) for $t \geq 0$ except possibly for $t=k h(k \in \mathbb{N})$.

Remark 5 As in the neutral case, the existence and uniqueness of a regular solution and a (continuous) concatenated solution can be confirmed in the retarded case too; uniqueness follows as in the arguments in Remark 4 while existence will be established in the following Theorems 3 and 4 , respectively. It is again true that if a regular solution exists, then it is a (continuous) concatenated solution at the same time. 
4 Conversion Method through the Output of $F$ for Regular and Pseudo Concatenated Solutions

In this section, we confine ourselves to the case in which $F$ in the time-delay feedback system $\Sigma$ is given the form

$$
\begin{gathered}
\dot{q}(t)=A q(t)+\left[B_{1} \cdots B_{\eta}\right]\left[\begin{array}{c}
u_{1}(t) \\
\vdots \\
u_{\eta}(t)
\end{array}\right] \\
{\left[\begin{array}{c}
y_{1}(t) \\
y_{2}(t) \\
\vdots \\
y_{\eta}(t)
\end{array}\right]=\left[\begin{array}{c}
I_{n} \\
0 \\
\vdots \\
0
\end{array}\right] q(t)+\left[\begin{array}{cccc}
D_{1} & \cdots & \cdots & D_{\eta} \\
I_{n} & 0 & \cdots & 0 \\
& \ddots & \ddots & \vdots \\
0 & & I_{n} & 0
\end{array}\right]\left[\begin{array}{c}
u_{1}(t) \\
u_{2}(t) \\
\vdots \\
u_{\eta}(t)
\end{array}\right]}
\end{gathered}
$$

where $q(t), u_{i}(t), y_{i}(t) \in \mathbb{R}^{n}(i=1, \cdots, \eta)$, and thus $B_{i}, D_{i} \in \mathbb{R}^{n \times n}(i=1, \cdots, \eta)$. The above $F$ is denoted by $F^{\mathrm{I}}$, and the corresponding $\Sigma$ is denoted by $\Sigma^{\mathrm{I}}$ in the following. The input/output relation of the delay $H$ is described by

$$
\left[\begin{array}{c}
u_{1}(t) \\
\vdots \\
u_{\eta}(t)
\end{array}\right]=\left[\begin{array}{c}
y_{1}(t-h) \\
\vdots \\
y_{\eta}(t-h)
\end{array}\right], \quad t \geq h
$$

Roughly speaking, what is established in this section is that the regular and pseudo concatenated solutions of the DDE (1) can be represented with the time-delay feedback system $\Sigma^{\mathrm{I}}$, provided that $A, B_{i}$ and $D_{i}(i=1, \cdots, \eta)$ are determined appropriately.

\subsection{Determining the Matrices in $F^{\mathrm{I}}$}

We first describe the state transition of the time-delay feedback system $\Sigma^{\mathrm{I}}$ by a DDE, and compare it with the DDE (1); the purpose of this subsection is to determine the matrices in $F^{\mathrm{I}}$ by comparing the matrices in these two DDEs. By such an approach, we can, in principle, have a conversion method of (1) into the time-delay feedback system $\Sigma^{\mathrm{I}}$. The result is very important in applying the monodromy operator approach to the DDE (1), and might be useful also for numerically computing the solution of (1).

It follows from (9) and (10) that

$$
\begin{aligned}
y_{1}(t) & =q(t)+D_{1} y_{1}(t-h)+\cdots+D_{\eta} y_{\eta}(t-h) \\
y_{i}(t) & =y_{i-1}(t-h), \quad i=2, \cdots, \eta
\end{aligned}
$$

It is immediate from (12) that

$$
y_{i}(t)=y_{1}(t-(i-1) h), \quad i=2, \cdots, \eta
$$

and substituting the above into (11) yields

$$
y_{1}(t)=q(t)+D_{1} y_{1}(t-h)+\cdots+D_{\eta} y_{1}(t-\eta h)
$$

or equivalently

$$
q(t)=y_{1}(t)-\sum_{i=1}^{\eta} D_{i} y_{1}(t-i h)
$$

Here, if we suppose that $y_{1}(t)$ is (continuous and) differentiable for $t \geq-\eta h$, then $q(t)$ is (con- 
tinuous and) differentiable for $t \geq 0$, and we have

$$
\dot{q}(t)=\dot{y}_{1}(t)-\sum_{i=1}^{\eta} D_{i} \dot{y}_{1}(t-i h)
$$

where $\dot{q}(t)$ and $\dot{y}_{1}(t-\eta h)$ denote, for $t=0$, the right-derivatives $\dot{q}(+0)$ and $\dot{y}_{1}(-\eta h+0)$, respectively. Substituting (10), (15) and (16) into (8) and rearranging the result lead to

$$
\dot{y}_{1}(t)=A y_{1}(t)+\sum_{i=1}^{\eta} D_{i} \dot{y}_{1}(t-i h)+\sum_{i=1}^{\eta}\left(B_{i}-A D_{i}\right) y_{1}(t-i h)
$$

By comparing this with (1), we see that if

$$
A=J, D_{i}=K_{i}, B_{i}=L_{i}+J K_{i}(i=1, \cdots, \eta)
$$

in (8) and (9), then $y_{1}(t)$ satisfies (1). This suggests that the solutions of the DDE (1) could be represented as the output $y_{1}(t)$ of the FDLTI system $F^{\mathrm{I}}$ in the time-delay feedback system $\Sigma^{\mathrm{I}}$. Before we can validate this observation, however, we need to note the following issues:

- When we view the above arguments as dealing with the response of $\Sigma^{\mathrm{I}}$ under the prescribed initial condition given by $q(0)$ and $u_{i}(t)(0 \leq t<h, i=1, \cdots, \eta)$, we must carefully observe that (11) and (12) hold in fact for $t \geq h,(13)$ for $t \geq(i-1) h$, and (14) and (15) for $t \geq \eta h$ only; thus the DDE (17) in fact holds only for $t \geq \eta h$, rather than $t \geq 0$. In other words, the above arguments do not take the initial condition of the DDE (1) into account, and thus it is not clear enough if the output $y_{1}(t)$ of $\Sigma^{\mathrm{I}}$ could really be made identical with the solution $x(t)$ of the DDE (1) by giving some appropriate initial condition for $\Sigma^{\mathrm{I}}$.

- $y_{1}(t)$ is assumed to be differentiable, but it has not been confirmed whether this assumption is satisfied.

These two issues are mutually related rather deeply, and will be resolved in the following subsection.

\subsection{Existence of the Regular and Pseudo Concatenated Solutions of DDE and Their Representation by $\Sigma^{\mathrm{I}}$}

We have the following two theorems on the regular and pseudo concatenated solutions of the neutral DDE (1) under the initial conditions (2) and (3). The proof is given in Appendix.

Theorem 1 Suppose that $\phi(t)$ is continuously differentiable on the interval $[-\eta h, 0]$ and satisfies $^{1}$

$$
\begin{aligned}
& \phi(0)=\xi \\
& \dot{\phi}(-0)=J \phi(0)+\sum_{i=1}^{\eta} K_{i} \dot{\phi}(-i h)+\sum_{i=1}^{\eta} L_{i} \phi(-i h)
\end{aligned}
$$

Then, the neutral DDE (1) has a (unique) regular solution $x(t)$, and it coincides, over $t \geq 0$, with $y_{1}(t)$ resulting from $\Sigma^{\mathrm{I}}$ with $F^{\mathrm{I}}$ given by (18) and with the initial state and initial input of $F^{\mathrm{I}}$ given respectively by

$$
q(0)=\xi-\sum_{i=1}^{\eta} K_{i} \phi(-i h)
$$

${ }^{1} \dot{\phi}(-0)$ denotes the left-derivative of $\phi(t)$ at $t=0$. Also, for notational convenience, the right-derivative of $\phi(t)$ at $t=\eta h$, i.e., $\dot{\phi}(-\eta h+0)$, is denoted simply by $\dot{\phi}(-\eta h)$. 
and

$$
u_{i}(t)=\phi(t-i h), \quad 0 \leq t<h, \quad i=1, \cdots, \eta
$$

Remark 6 The first assertion (on the existence of a regular solution) can be found, e.g, in Bellman and Cooke (1963); the condition (20) is called the splicing condition or the sewing condition (Bellen and Zennaro 2003, Kolmanovskii and Myshkis 1999, Kolmanovskii and Nosov 1986), whose necessity is obvious. The most important assertion of the above theorem, however, is the latter assertion that a solution of the DDE (1) can be represented with a suitably determined time-delay feedback system $\Sigma$. It also is a unique feature of the present paper that the first assertion is proved in an explicit relationship with the latter assertion.

Theorem 2 Suppose that $\phi(t)$ is bounded and continuously differentiable on each of the intervals $[-i h,-(i-1) h)(i=1, \cdots, \eta)$. Then, the neutral DDE (1) has a (unique) pseudo concatenated solution $x(t)$, and it coincides, over $t \geq 0$, with $y_{1}(t)$ resulting from $\Sigma^{\mathrm{I}}$ with $F^{\mathrm{I}}$ given by (18) and with the initial state and initial input of $F^{\mathrm{I}}$ given by (21) and (22), respectively.

\subsection{Illustrative Examples for the Neutral Case}

We take a simple neutral DDE (also found in Kolmanovskii and Myshkis (1999)) with different initial conditions, and illustrate the above theorems. We begin with an example pertinent to Theorem 1.

Example 1 Consider the neutral DDE

$$
\dot{x}(t)=\dot{x}(t-h)
$$

which corresponds to $\eta=1, K_{1}=1, L_{1}=0$ and $J=0$. Let the initial conditions be $\phi(t)=$ $1(-h \leq t \leq 0)$ and $\xi=\phi(0)$. Then, the conditions of Theorem 1 are satisfied; the corresponding $F^{\mathrm{I}}$ is given by

$$
\dot{q}(t)=0, \quad y_{1}(t)=q(t)+u_{1}(t)
$$

and the initial conditions are given by $q(0)=0$ and $u_{1}(t)=1(0 \leq t<h)$. This leads to $y_{1}(t)=1(t \geq 0)$. On the other hand, we can readily verify that (23) has a regular solution $x(t)=1(t \geq-h)$ under the above initial conditions, which we see is coincident, over $t \geq 0$, with the above $y_{1}(t)$ resulting from $\Sigma^{\mathrm{I}}$. Hence, the validity of the assertion of Theorem 1 can be verified.

We next give two examples pertinent to Theorem 2 .

Example 2 Consider again the DDE (23) but with the initial conditions $\phi(t)=0(-h \leq t \leq 0)$ and $\xi=1(\neq \phi(0))$. Then, the hypotheses of Theorem 1 are not fulfilled, but Theorem 2 may be applied instead. The resulting $\Sigma^{\mathrm{I}}$ remains the same as that in Example 1, and the initial state and initial input of $F^{\mathrm{I}}$ are given by $q(0)=1$ and $u_{1}(t)=0(0 \leq t<h)$, respectively. This leads to $y_{1}(t)=k((k-1) h \leq t<k h)$ over $t \geq 0$. Hence, Theorem 2 asserts that the modified form of (23) given by

$$
\frac{d}{d t} v(t)=\frac{d}{d t}[x(t)-x(t-h)]=0
$$

has a concatenated solution (equivalently, (23) has a (unique) pseudo concatenated solution) $x(t)=k((k-1) h \leq t<k h, k \in \mathbb{N})$ over $t \geq 0$. We can readily see that this $x(t)$, supplemented

\footnotetext{
${ }^{1}$ Unlike in the situation of Theorem 1 , the state $q(t)$ of $F^{\mathrm{I}}$ is not necessarily differentiable at $t=k h(k \in \mathbb{N})$; that is, (8) does not have a differentiable solution, in general. However, there does exist a (unique) continuous (and "piecewise differentiable") function $q(t)$ satisfying (8) except possibly for $t=k h(k \in \mathbb{N})$, and $y_{1}(t)$ in this theorem refers to the one that corresponds to such $q(t)$. The same remark applies also to other similar theorems in the following (i.e., Theorems 4,6 and 8).
} 
with $x(t)=\phi(t)=0(-h \leq t<0)$, is indeed a pseudo concatenated solution of (23) if we note that $v(t)=x(t)-x(t-h)=1(t \geq 0)$; it is continuous and differentiable over $t \geq 0$ and satisfies (25). However, $x(t)$ itself is not continuous at $t=k h(k \in \mathbb{N})$, and thus is only a (unique) pseudo concatenated solution of (23).

Example 3 Consider once again the DDE (23) but with the initial conditions $\phi(t)=\sin ((\pi / h) t)$ $(=-\sin ((\pi / h) \tau)),-h \leq t=-h+\tau \leq 0$ and $\xi=\phi(0)$; contrary to Example 2, this example corresponds to the case in which (19) is satisfied but (20) is not. Following Theorem 2, we give the initial state and initial input of $F^{\mathrm{I}}$ by $q(0)=0$ and $u_{1}(t)=-\sin ((\pi / h) t), 0 \leq t<h$, respectively. Then, we see that

$$
y_{1}(t)=-\sin ((\pi / h) \tau), t=k h+\tau, 0 \leq \tau<h(k=0,1, \cdots)
$$

Letting $x(t)=y_{1}(t)$ for $t \geq 0$ and $x(t)=\phi(t)$ for $-h \leq t<0$ lead to

$$
x(t)=-\sin ((\pi / h) \tau), t=k h+\tau, 0 \leq \tau<h(k=-1,0,1, \cdots)
$$

We can verify that the resulting $v(t)=x(t)-x(t-h)$ is identically zero for $t \geq 0$ and thus is continuous, differentiable and satisfies (25) for $t \geq 0$. However, $x(t)$ itself is not differentiable at $t=k h(k \in \mathbb{N})$, and thus is only a (unique) pseudo concatenated solution of (23) (rather than a regular solution).

\subsection{The Retarded Case}

For the retarded DDE (7), we have the following two theorems corresponding to Theorems 1 and 2, respectively. Again, the proof is given in Appendix.

Theorem 3 Suppose that $\phi(t)$ is continuous on the interval $[-\eta h, 0]$ and satisfies (19). Then, the retarded $D D E(7)$ has a (unique) regular solution $x(t)$, and it coincides, over $t \geq 0$, with $q(t)$ resulting from $\Sigma^{\mathrm{I}}$ with $F^{\mathrm{I}}$ given by (18) and with the initial state and initial input of $F^{\mathrm{I}}$ given by $q(0)=\xi(=\phi(0))$ and (22), respectively.

Remark 7 The first assertion (on the existence of a regular solution) can be found, e.g., in Hale and Lunel (1993, Theorem 2.1 (p. 14)), and the importance of this theorem lies in the latter assertion. However, the derivation of the first assertion here would also be unique in the sense that it is through such arguments relevant to the latter assertion.

Theorem 4 Suppose that $\phi(t)$ is bounded and continuous on each of the intervals $[-i h,-(i-$ 1)h) $(i=1, \cdots, \eta)$. Then, the retarded DDE (7) has a (unique) continuous concatenated solution $x(t)$, and it coincides, over $t \geq 0$, with $(t)$ resulting from $\Sigma^{\mathrm{I}}$ with $F^{\mathrm{I}}$ given by (18) and with the initial state and initial input of $F^{\mathrm{I}}$ given by $q(0)=\xi$ and (22), respectively.

\subsection{Illustrative Examples for the Retarded Case}

Example 4 Consider the retarded DDE

$$
\dot{x}(t)=x(t-h)
$$

which corresponds to $\eta=1, L_{1}=1, J=0$. Let the initial conditions be $\phi(t)=1(-h \leq t \leq 0)$ and $\xi=\phi(0)$. If we view (28) as a special form of the neutral DDE (1) and consider applying Theorem 1, then (20) is not satisfied and thus the existence of a regular solution cannot be concluded. However, we can apply Theorem 3 instead, which asserts the existence of a regular solution and its coincidence (over $t \geq 0$ ) with $q(t)$ resulting from $\Sigma^{\mathrm{I}}$ with $F^{\mathrm{I}}$ given by

$$
\dot{q}(t)=u_{1}(t), \quad y_{1}(t)=q(t)
$$

and the initial conditions $q(0)=1, u_{1}(t)=1(0 \leq t<h)$. By solving the above differential equation together with (10), we see that $x(t)$ is given by $x(t)=q(t)=1+t$ for $0 \leq t<h$, and 
by $x(t)=q(t)=1+\frac{1}{2} h^{2}+(1-h) t+\frac{1}{2} t^{2}$ for $h \leq t<2 h$, and so on (which we can confirm is indeed a regular solution of (28)). Hence, we see that the regular solution $x(t)$ is differentiable at $t=h$ but not (two-side) differentiable at $t=0$. This can be interpreted in correspondence with the fact that the definition of a regular solution for a retarded DDE is free from the requirement on the (two-side) differentiability at $t=0$; see Remark 2.

Example 5 Consider again the DDE (28) but with the initial conditions $\phi(t)=0(-h \leq t \leq 0)$ and $\xi=1(\neq \phi(0))$. Then, the hypotheses of Theorem 3 are not fulfilled (more precisely, (19) is not satisfied), but Theorem 4 may be applied instead. The resulting $\Sigma^{\mathrm{I}}$ remains the same as that in Example 4, and the initial state and initial input of $F^{\mathrm{I}}$ are given by $q(0)=1$ and $u_{1}(t)=0(0 \leq t<h)$, respectively. This leads to $q(t)=1$ for $0 \leq t<h$, and $q(t)=1-h+t$ for $h \leq t<2 h$, and so on. Letting $x(t)=q(t), 0 \leq t<2 h$, we can confirm that it, supplemented with the initial function, indeed satisfies (28) except for $t=h$. However, it is not differentiable at $t=h$. That is, this solution is not a regular solution but is only a (continuous) concatenated solution.

Example 6 We next consider an example with discontinuous $\phi(t)$. Let us consider $\eta=2$, $L_{1}=0, L_{2}=1, J=0$, i.e., the retarded DDE given by

$$
\dot{x}(t)=x(t-2 h)
$$

with the initial conditions given by $\phi(t)=0(-2 h \leq t<h), \phi(t)=1(-h \leq t<0)$ and $\xi=2$. The resulting $F^{\mathrm{I}}$ in $\Sigma^{\mathrm{I}}$ is given by

$$
\dot{q}(t)=\left[\begin{array}{ll}
0 & 1
\end{array}\right]\left[\begin{array}{l}
u_{1}(t) \\
u_{2}(t)
\end{array}\right], y_{1}(t)=q(t), y_{2}(t)=u_{1}(t)
$$

with the initial state and initial input given by $q(0)=2$ and $u_{1}(t)=1, u_{2}(t)=0(\leq t<h)$, respectively. Hence, we have

$$
q(t)= \begin{cases}2 & (0 \leq t<h) \\ 2-h+t & (h \leq t<2 h) \\ 2-3 h+2 t & (2 h \leq t<3 h) \\ \cdots & \end{cases}
$$

Letting $x(t)=q(t), t \geq 0$, it, supplemented with the initial function, is continuous, and is differentiable and satisfies (30) except for $t=h, 2 h$. This is indeed a continuous concatenated solution of (30).

\section{Conversion Method through the State of $\boldsymbol{F}$ for Regular and Continuous Concatenated Solutions}

In this section, we confine ourselves to the case in which $F$ in the time-delay feedback system $\Sigma$ is given the form

$$
\begin{gathered}
\dot{q}(t)=A q(t)+\left[\begin{array}{llll}
I_{n} & 0 & \cdots & 0
\end{array}\right]\left[\begin{array}{c}
u_{1}(t) \\
u_{2}(t) \\
\vdots \\
u_{\eta}(t)
\end{array}\right] \\
{\left[\begin{array}{c}
y_{1}(t) \\
y_{2}(t) \\
\vdots \\
y_{\eta}(t)
\end{array}\right]=\left[\begin{array}{c}
C_{1} \\
C_{2} \\
\vdots \\
C_{\eta}
\end{array}\right] q(t)+\left[\begin{array}{cccc}
D_{1} & I_{n} & & 0 \\
D_{2} & 0 & \ddots & \\
\vdots & \vdots & \ddots & I_{n} \\
D_{\eta} & 0 & \cdots & 0
\end{array}\right]\left[\begin{array}{c}
u_{1}(t) \\
u_{2}(t) \\
\vdots \\
u_{\eta}(t)
\end{array}\right]}
\end{gathered}
$$


where $q(t), u_{i}(t), y_{i}(t) \in \mathbb{R}^{n}(i=1, \cdots, \eta)$, and thus $C_{i}, D_{i} \in \mathbb{R}^{n \times n}(i=1, \cdots, \eta)$. The above $F$ is denoted by $F^{\mathrm{II}}$, and the corresponding $\Sigma$ is denoted by $\Sigma^{\mathrm{II}}$ in the following. Roughly speaking, what is established in this section is that the regular and continuous concatenated solutions of the DDE (1) can be represented with the time-delay feedback system $\Sigma^{\mathrm{II}}$, provided that $A, C_{i}$ and $D_{i}(i=1, \cdots, \eta)$ are determined appropriately.

\subsection{Determining the Matrices in $\mathrm{F}^{\mathrm{II}}$}

We first describe the state transition of the time-delay feedback system $\Sigma^{\mathrm{II}}$ by a DDE, and compare it with the DDE (1). We can determine the matrices in $F^{\mathrm{II}}$ in this way, which can be used for converting (1) into the time-delay feedback system $\Sigma^{\mathrm{II}}$. Such conversion is very important in applying the monodromy operator approach to the DDE (1), and might be useful also for numerically computing the solution of (1).

It follows from (33) that

$$
u_{1}(t)=\dot{q}(t)-A q(t)
$$

We also have

$$
\begin{aligned}
& u_{i}(t)=C_{i} q(t-h)+D_{i} u_{1}(t-h)+u_{i+1}(t-h) \quad(i=1, \cdots, \eta-1) \\
& u_{\eta}(t)=C_{\eta} q(t-h)+D_{\eta} u_{1}(t-h)
\end{aligned}
$$

from (34) if we note (10). Substituting (35) into the above equations leads to

$$
\begin{aligned}
& u_{i}(t)=D_{i} \dot{q}(t-h)+u_{i+1}(t-h)+\left(C_{i}-D_{i} A\right) q(t-h) \quad(i=1, \cdots, \eta-1) \\
& u_{\eta}(t)=D_{\eta} \dot{q}(t-h)+\left(C_{\eta}-D_{\eta} A\right) q(t-h)
\end{aligned}
$$

Hence, we readily have

$$
\begin{gathered}
u_{i}(t)=\sum_{k=i}^{\eta} D_{k} \dot{q}(t-(k-i+1) h)+\sum_{k=i}^{\eta}\left(C_{k}-D_{k} A\right) q(t-(k-i+1) h) \\
(i=1, \cdots, \eta)
\end{gathered}
$$

Substituting the above equation with $i=1$ into (33) leads to the DDE

$$
\dot{q}(t)=A q(t)+\sum_{i=1}^{\eta} D_{i} \dot{q}(t-i h)+\sum_{i=1}^{\eta}\left(C_{i}-D_{i} A\right) q(t-i h)
$$

Comparison of this DDE with (1) suggests that if

$$
A=J, C_{i}=L_{i}+K_{i} J, D_{i}=K_{i} \quad(i=1, \cdots, \eta)
$$

in $F^{\mathrm{II}}$, then the solution $x(t)$ of the DDE (1) could be represented as the state $q(t)$ of the FDLTI system $F^{\mathrm{II}}$ in the time-delay feedback system $\Sigma^{\mathrm{II}}$.

\subsection{Existence of the Regular and Continuous Concatenated Solutions of DDE and Their Representation by $\Sigma^{\mathrm{II}}$}

Suppose that the initial state and initial input of $F^{\mathrm{II}}$ in $\Sigma^{\mathrm{II}}$ are given respectively by

$$
q(0)=\xi
$$

and

$$
\begin{gathered}
u_{i}(t)=\sum_{k=i}^{\eta} K_{k} \dot{\phi}(t-(k-i+1) h)+\sum_{k=i}^{\eta} L_{k} \phi(t-(k-i+1) h), \\
0 \leq t<h \quad(i=1, \cdots, \eta)
\end{gathered}
$$


Under these initial conditions, we have the following two theorems regarding the regular and continuous concatenated solutions of the neutral DDE (1). In particular, the first one corresponds to an alternative result to Theorem 1 for representing the same type of solution (i.e., a regular solution) with a different time-delay feedback system $\Sigma$.

Theorem 5 Suppose that $\phi(t)$ is continuously differentiable on $[-\eta h, 0]$ and satisfies (19) and (20). Then, the neutral DDE (1) has a (unique) regular solution $x(t)$, and it coincides, over $t \geq 0$, with $q(t)$ resulting from $\Sigma^{\mathrm{II}}$ with $F^{\mathrm{II}}$ given by (42) and with the initial state and initial input of $F^{\mathrm{II}}$ given by (43) and (44), respectively.

Theorem 6 Suppose that $\phi(t)$ is bounded and continuously differentiable on each of the intervals $[-i h,-(i-1) h)(i=1, \cdots, \eta)$ and has limits $\lim _{t \rightarrow-(i-1) h-0} \phi(t)(i=1, \cdots, \eta)$. Then, the neutral DDE (1) has a (unique) continuous concatenated solution $x(t)$, and it coincides, over $t \geq 0$, with $(t)$ resulting from $\Sigma^{\mathrm{II}}$ with $F^{\mathrm{II}}$ given by (42) and with the initial state and initial input of $F^{\mathrm{II}}$ given by (43) and (44), respectively.

If we recall Theorems 1 and 2 in the preceding section, we see that the above two theorems are parallel results to them. It should be noted, however, that the theorems in this section represent the regular and continuous concatenated solutions of the DDE (1) with the state of $F$, while the theorems in the preceding section represent the regular and pseudo concatenated solutions of the DDE (1) with the output of $F$. Due to this difference, together with the fact that a pseudo concatenated solution is allowed to be discontinuous, the theorems in the preceding section are free from evaluating the derivative of $\phi(t)$ to determine the initial input to $F$, and they hold under a slightly weaker assumption on $\phi(t)$.

On the other hand, a continuous concatenated solution $x(t)$ of the DDE (1) must be continuous by definition, and thus $\lim _{t \rightarrow k h-0} x(t)(k \in \mathbb{N})$ must exist. The additional hypothesis in Theorem 6 on the existence of $\lim _{t \rightarrow-(i-1) h-0} \phi(t)(i=1, \cdots, \eta)$ corresponds to this requirement, and also ensures the existence of $\lim _{t \rightarrow k h-0} q(t)$ in $\Sigma^{\mathrm{II}}$; in showing the existence of the latter, the derivative of $\phi(t)$ that appears in (44) can be handled easily with the integration by parts under this hypothesis ${ }^{1}$. The details of the proofs of the above theorems are omitted, which are parallel to those of the preceding theorems.

\subsection{Illustrative Examples for the Neutral Case}

We study three examples that are parallel to those studied in the preceding section.

Example 7 Consider the same neutral DDE as in Example 1, i.e., $\dot{x}(t)=\dot{x}(t-h)$, where we take the same initial conditions, i.e., $\phi(t)=1(-h \leq t \leq 0)$ and $\xi=\phi(0)$. The corresponding $F^{\mathrm{II}}$ is given by

$$
\dot{q}(t)=u_{1}(t), \quad y_{1}(t)=u_{1}(t)
$$

with the initial state and initial input given by $q(0)=1$ and $u_{1}(t)=0(0 \leq t<h)$, respectively. Hence, we have $q(t)=1(t \geq 0)$, and thus Theorem 5 asserts that the regular solution of (45) is given by $x(t)=1(t \geq-h)$. This assertion is consistent with that in Example 1 and its validity has been confirmed there.

Example 8 Consider $\dot{x}(t)=\dot{x}(t-h)$ again but with the initial conditions replaced by those in Example 2, i.e., $\phi(t)=0(-h \leq t \leq 0)$ and $\xi=1(\neq \phi(0))$. Then, the hypotheses of Theorem 5 are not fulfilled, but Theorem 6 may be applied instead. The resulting $\Sigma^{\mathrm{II}}$ remains the same as that in Example 7, and the initial state and initial input are also the same as in Example 7, i.e.,

${ }^{1}$ In Theorem 8 given below for retarded DDEs, we do not have to deal with $\dot{\phi}(t)$ in $(44)$ because of $K_{i}=0(i=1, \cdots, \eta)$. Hence, no such additional hypothesis arises in that theorem. 
$q(0)=1$ and $u_{1}(t)=0(0 \leq t<h)$. Hence, Theorem 6 asserts that the continuous concatenated solution of the neutral $D D E \dot{x}(t)=\dot{x}(t-h)$ is given by

$$
x(t)=\left\{\begin{array}{l}
0(-h \leq t<0) \\
1(t \geq 0)
\end{array}\right.
$$

We can indeed verify that the above $x(t)$ is the solution that satisfies the requirements in Definition 3. This solution, however, is different from the pseudo concatenated solution provided in Example 2 for the same DDE under the same initial conditions. This demonstrates that the neutral DDE (1) with initial conditions failing to satisfy the hypothesis of Theorem 1 (or equivalently, Theorem 5) generally has different "non-regular" solutions, according to different definitions for its solutions.

Example 9 Consider once again the DDE $\dot{x}(t)=\dot{x}(t-h)$ but with the initial conditions replaced by those studied in Example 3, i.e., $\phi(t)=\sin ((\pi / h) t),-h \leq t \leq 0$ and $\xi=\phi(0)$. We can then apply Theorem 6 (but not Theorem 5). We have the same $F^{\mathrm{II}}$ as in (45) with the initial state $q(0)=0$ and the initial input $u_{1}(t)=-\cos ((\pi / h) t), 0 \leq t<h$, and it thus follows that

$$
q(t)=-\sin ((\pi / h) \tau), t=k h+\tau, 0 \leq \tau<h(k=0,1, \cdots)
$$

Theorem 6 asserts that this coincides with the continuous concatenated solution for $t \geq 0$. That is, by supplementing the initial condition, it asserts that the continuous concatenated solution for $t \geq-h$ is given by

$$
x(t)=-\sin ((\pi / h) \tau), t=k h+\tau, 0 \leq \tau<h(k=-1,0,1, \cdots)
$$

We can indeed verify that the above $x(t)$ is the solution that satisfies the requirements in Definition 3. It turns out that this continuous concatenated solution actually coincides with the pseudo concatenated solution provided in Example 3 for the same DDE under the same initial conditions.

We have seen in the above example that, under some initial conditions, a pseudo concatenated solution of (1) (that is not a regular solution) can possibly be a continuous concatenated solution at the same time. Regarding such coincidence, we have the following result.

Proposition 1 Suppose that $\phi(t)$ is bounded and continuously differentiable on each of the intervals $[-i h,-(i-1) h)(i=1, \cdots, \eta)$ and has the limits $\phi_{i}:=\lim _{t \rightarrow-i h-0} \phi(t)(i=0, \cdots, \eta-1)$. Let $\xi_{0}:=\xi$ and $\xi_{i}:=\phi(-i h)(i=1, \cdots, \eta-1)$, and define $\delta_{i}:=\xi_{i}-\phi_{i}(i=0, \cdots, \eta-1)$. Then, the continuous concatenated solution and pseudo concatenated solution of (1) coincide with each other if and only if

$$
\sum_{i=k}^{\eta} K_{i} \delta_{i-k}=0, \quad k=1, \cdots, \eta
$$

Proof We first remark that the continuous and pseudo concatenated solutions of (1) exist and are unique under the hypotheses of this proposition (Remark 4).

(Sufficiency) The pseudo concatenated solution $x(t)$ can be written as $x(t)=v(t)+$ $\sum_{i=1}^{\eta} K_{i} x(t-i h)$. Since $v(t)$ is right-continuous at $t=0$ by definition, it is obvious from the hypothesis on $\phi(t)$ that this $x(t)$ is right-continuous at $t=0$. Next, since $v(t)$ is continuous at $t=h$ again by definition, the pseudo concatenated solution $x(t)$ is continuous at $t=h$ if the condition (49) holds for $k=1$. In a similar fashion, we can establish the continuity of the pseudo concatenated solution $x(t)$ at $t=k h, k=2, \cdots, \eta$ by the condition (49) for $k=2, \cdots, \eta$. Using the continuity thus established, together with the relation $x(t)=v(t)+\sum_{i=1}^{\eta} K_{i} x(t-i h)$ again, we can readily establish the continuity of $x(t)$ at $t=(\eta+1) h$, and by repeating this procedure,

\footnotetext{
${ }^{1} \delta_{i}$ corresponds to the jump of the initial function $x(t),-\eta h \leq t \leq 0$ of the DDE (1) at its possible discontinuity points
} $t=-i h(i=0, \cdots, \eta-1)$. 
we can establish the continuity of the pseudo concatenated solution $x(t)$ over $t \geq 0$. Hence, we can readily verify that $x(t), t \geq-h$ satisfies the requirements in Definition 3 , and thus it is the continuous concatenated solution of (1) at the same time.

(Necessity) It is easy to see from similar arguments to the above that the pseudo concatenated solution $x(t)$ is discontinuous at $t=k h$ for the smallest $k$ that fails to meet the condition (49). It is obvious that such a discontinuous solution is not a continuous concatenated solution.

Remark 8 It is very often the case in the literature that the initial functions for the neutral DDE (1) are assumed to be continuously differentiable on the interval $[-\eta h, 0]$. This condition alone is not enough to ensure the existence of a regular solution of (1) (recall Theorems 1 and 5), and thus a definition of a solution is necessary that is more general than that of a regular solution. Regarding the two possible definitions for such a purpose, i.e., a pseudo concatenated solution and a continuous concatenated solution, however, their mutual distinction is not necessary under the above usual assumption on the initial functions. This is because all the conditions in Proposition 1 are satisfied automatically under this assumption and thus these two types of generalized solutions coincide with each other after all; it seems that it is because of this situation that the distinct definitions for pseudo and continuous concatenated solutions are scarcely found in the literature.

\subsection{The Retarded Case}

When confined to the retarded DDE (7), we have the following two theorems corresponding to Theorems 5 and 6 , respectively.

Theorem 7 Suppose that $\phi(t)$ is continuous on the interval $[-\eta h, 0]$ and satisfies (19). Then, the retarded $D D E(7)$ has a (unique) regular solution $x(t)$, and it coincides, over $t \geq 0$, with $q(t)$ resulting from $\Sigma^{\mathrm{II}}$ with $F^{\mathrm{II}}$ given by (42) and with the initial state and initial input of $F^{\mathrm{II}}$ given by $q(0)=\xi(=\phi(0))$ and (44), respectively.

Theorem 8 Suppose that $\phi(t)$ is bounded and continuous on each of the intervals $[-i h,-(i-$ 1) $h)(i=1, \cdots, \eta)$. Then, the retarded DDE (7) has a (unique) continuous concatenated solution $x(t)$, and it coincides, over $t \geq 0$, withq $(t)$ resulting from $\Sigma^{\mathrm{II}}$ with $F^{\mathrm{II}}$ given by (42) and with the initial state and initial input of $F^{\mathrm{II}}$ given by $q(0)=\xi$ and (44), respectively.

\subsection{Illustrative Examples for the Retarded Case}

Example 10 Consider the same retarded DDE as in Example 4, i.e., $\dot{x}(t)=x(t-h)$, where we take the same initial conditions, i.e., $\phi(t)=1(-h \leq t \leq 0)$ and $\xi=\phi(0)$. The corresponding $F^{\mathrm{II}}$ is given by

$$
\dot{q}(t)=u_{1}(t), \quad y_{1}(t)=q(t)
$$

with the initial state and initial input given by $q(0)=1$ and $u_{1}(t)=1(0 \leq t<h)$, respectively. This situation is exactly the same as that in Example 4, and hence we can confirm that we arrive at the same conclusion as in that example.

Example 11 Consider $\dot{x}(t)=x(t-h)$ again but with the initial conditions replaced by those in Example 5, i.e., $\phi(t)=0(-h \leq t \leq 0)$ and $\xi=1(\neq \phi(0))$. Then, the hypotheses of Theorem 7 are not fulfilled (more precisely, (19) is not satisfied), but Theorem 8 may be applied instead. The resulting $\Sigma^{\mathrm{II}}$ remains the same as that in Example 10, and thus coincides with $\Sigma^{\mathrm{I}}$ in Example 5. Furthermore, the initial state $q(0)=1$ and the initial input $u_{1}(t)=0(\leq t<h)$ of $F^{\mathrm{II}}$ are also the same as those for $F^{\mathrm{I}}$ in Example 5. Hence, we can confirm that we arrive at the same conclusion as in that example.

Example 12 Consider the same retarded $D D E \dot{x}(t)=x(t-2 h)$ as in Example 6 , as well as the same discontinuous initial function given by $\phi(t)=0(-2 h \leq t<h), \phi(t)=1(-h \leq t<0)$ 
and $\xi=2$. The resulting $F^{\mathrm{II}}$ in $\Sigma^{\mathrm{II}}$ is given by

$$
\dot{q}(t)=\left[\begin{array}{ll}
1 & 0
\end{array}\right]\left[\begin{array}{l}
u_{1}(t) \\
u_{2}(t)
\end{array}\right], y_{1}(t)=u_{2}(t), y_{2}(t)=q(t)
$$

with the initial state $q(0)=2$ and the initial input $u_{1}(t)=0$ and $u_{2}(t)=1(0 \leq t<h)$. This situation is nothing but that in Example 4 with $u_{1}(t)$ and $u_{2}(t)$ exchanged with each other, and hence we can confirm that we arrive at the same conclusion as in that example.

In all of the above three examples about retarded DDEs, we have seen that the results with $\Sigma^{\mathrm{I}}$ and $\Sigma^{\mathrm{II}}$ coincide with each other. This is a natural consequence since there exists no distinction between pseudo concatenated solutions and continuous concatenated solutions in retarded DDEs (recall also Remark 5 about the uniqueness of solutions). It would be worthwhile to note that, unlike in the case of neutral DDEs, these two types of concatenated solutions coincide irrespective of the condition (49); we could interpret that this condition is always satisfied in retarded DDEs because of $K_{i}=0(i=1, \cdots, \eta)$.

\section{Conclusion}

This paper first gave the definitions for the solutions of the delay-differential equation (DDE) (1) under possibly discontinuous initial functions, and then studied the conversion problems of this DDE into the representation as a time-delay feedback system. We gave two such methods, each of which corresponds to dealing with the solution of (1) under a different definition, in general; pseudo concatenated solutions and continuous concatenated solutions. Examples illustrating the subtleties about the definitions of the solutions of (1) as well as the validity of the conversion methods were also provided.

Roughly speaking, the most important conclusion is that the same DDE can have two different types of solutions, depending on how the solutions of DDEs are defined, whereas each type of solutions is unique once we determine which definition of the solutions we adopt from the two alternatives. In this sense, the representation of time-delay systems (TDSs) as DDEs alone might leave a lot of ambiguity, in general, and thus the pair of a DDE and its solution definition should be considered. Once such a pair is given, we can have its equivalent representation as a timedelay feedback system $\Sigma$ (either as $\Sigma^{\mathrm{I}}$ or $\Sigma^{\mathrm{II}}$ ), where the solution of $\Sigma$ is always unique and the distinction between the two types of concatenated solutions in the DDE representation is absorbed into the differences in the structure of the time-delay system $\Sigma$ (i.e., $\Sigma^{\mathrm{I}}$ or $\Sigma^{\mathrm{II}}$ ) and its associated initial conditions. This suggests that the ability for representing TDSs is higher in the treatment as the time-delay feedback system $\Sigma$ than in the treatment as DDEs, which might be contrary to the general belief. This aspect can be supported further by studying the opposite direction of conversion (i.e., from the time-delay feedback system $\Sigma$ into a DDE); it turns out that such conversion is possible in a sense (i.e., if the conversion of the associated initial conditions were left untouched), but that we can have exactly parallel results to those in this paper only under some appropriate assumptions on the initial conditions of $\Sigma$. The details will be reported independently (Yamazaki and Hagiwara 2011), together with the extended results of this paper to the case of DDEs with external inputs and outputs. Naturally, the notions of pseudo and continuous concatenated solutions of DDEs play important roles in such studies, too. Possible further research directions include (i) the extension of these solution notions and the associated conversion problems to the case of time-varying and/or nonlinear TDSs, (ii) the introduction of further different types of solution notions of DDEs under possibly discontinuous initial conditions, and (iii) the study on the relevance among the different solution notions, the actual behavior of practical TDSs, and their relationship to the modeling procedures of such TDSs as DDEs. Another rather straightforward extension includes investigating, from the results derived in this paper, how smooth the solutions can be, depending on the smoothness of the initial functions, as well as the difference in the smoothing effect in the retarded case and the 
neutral case. Even though such results are well known, we believe that the arguments of this paper as a whole, in spite of its restriction to a simple class of DDEs, are also of pedagogical value and successful in giving an almost entire picture about the theoretical subtleties on the definitions and properties of the solutions of the DDE (1).

\section{References}

Baker, C. T. H., and Paul, C. A. H., 2006, Discontinuous solutions of neutral delay differential equations, Applied Numerical Mathematics, Vol. 56, pp. 284-304.

Bamieh, B. A., and Pearson, J. B., 1992, A general framework for linear periodic systems with applications to $H_{\infty}$ sampled-data control, IEEE Trans. Automat. Contr., Vol. 37, No. 4, pp. 418-435.

Bellen, A., and Zennaro, M., 2003, Numerical Methods for Delay Differential Equations, Oxford Science Publications.

Bellman, R., and Cooke, K. L., 1963, Differential-Difference Equations, Academic Press.

Hagiwara, T., 2008, Fast-lifting approach to time-delay systems: Fundamental framework, Proc. the 47th IEEE Conference on Decision and Control, pp. 5292-5299.

Hagiwara, T., 2010, Monodromy operator approach to time-delay systems: Fast-lifting based treatment of operator Lyapunov inequalities, 19th International Symposium on Mathematical Theory of Networks and Systems, pp. 1187-1194.

Hagiwara, T., and Fujinami, T., 2010, Fast-lifting approach to the computation of the spectral radius of neutral time-delay systems, 9th IFAC Workshop on Time Delay Systems, FP-HT-896.

Hagiwara, T., and Hirata, K., 2011, Fast-lifting approach to the computation of the spectrum of retarded time-delay systems, Proc. European Control Conference 2009, pp. 3575-3580; to appear in European Journal of Control.

Hagiwara, T., and Inui, T., 2010, Monodromy operator approach to time-delay systems: Numerical method for solving operator Lyapunov inequalities, 19th International Symposium on Mathematical Theory of Networks and Systems, 1195-1202.

Hale, J., 1977, Theory of Functional Differential Equations, Springer-Verlag.

Hale, J. K., and Lunel, S. M. V., 1993, Introduction to Functional Differential Equations, Springer-Verlag.

Hirata, K., and Kokame, H., 2003, Stability analysis of retarded systems via lifting technique, Proc. the 42nd IEEE Conference on Decision and Control, pp. 5595-5596.

Kolmanovskii, V., and Myshkis, A., 1999, Introduction to the Theory and Applications of Functional Differential Equations, Kluwer.

Kolmanovskii, V. B., and Nosov, V. R., 1986, Stability of Functional Differential Equations, Academic Press.

Krishna, S. V., and Anokhin, A. V., 1994, Delay differential systems with discontinuous initial data and existence and uniqueness theorems for systems with impulse and delay, J. Applied Mathematics and Stochastic Analysis, Vol. 7, No. 1, pp. 49-67.

Yamamoto, Y., 1994, A function space approach to sampled-data systems and tracking problems, IEEE Trans. Automat. Contr., Vol. AC-39, No. 4, pp. 703-713.

Yamazaki T., and Hagiwara, T., 2011, A note on mutual conversion between linear time-invariant delay-differential equations and time-delay feedback systems, to be presented at SICE Annual Conference 2011.

\section{Appendix A: Proofs of Theorems}

Proof of Theorem 1. We have seen in Section 4.1 that a solution of the DDE (1) is expected 
to be represented as the output $y_{1}(t)$ of $F^{\mathrm{I}}$ in $\Sigma^{\mathrm{I}}$. Hence, let us accordingly define

$$
y_{1}(t)=\phi(t), \quad-\eta h \leq t<0
$$

and view $y_{1}(t)$ to be defined not only for $t \geq 0$ but for $t \geq-\eta h$ in the following. It then follows immediately from $(22)$ that

$$
u_{i}(t)=y_{1}(t-i h), \quad 0 \leq t<h, i=1, \cdots, \eta
$$

and hence by (9), we see that (15) holds also for $0 \leq t<h$ (rather than only for $t \geq \eta h$ as remarked at the stage of Section 4.1). We can see readily from this, together with the arguments in Section 4.1, that if $y_{1}(t)$ is (continuous and) differentiable for $-\eta h \leq t<h$, then it satisfies (17) (and thus coincides with the solution $x(t)$ of (1)) for $0 \leq t<h$. Hence, the former half of the proof is devoted to showing that $y_{1}(t)$ indeed satisfies this differentiability condition.

It follows from (A1) that $y_{1}(t)$ is (continuous and) differentiable for $-\eta h \leq t<0$. Hence, we aim at showing that it is differentiable also for $-\eta h \leq t<h$ (in fact, it will turn out that it is continuously differentiable there). It follows from $(22)$ that $\left[u_{1}(t)^{T}, \cdots, u_{\eta}(t)^{T}\right]^{T}$ is continuous for $0 \leq t<h$, and thus from (8), q(t), $t \geq 0$ is continuously differentiable for $0 \leq t<h$. Hence, by (15), $y_{1}(t)$ is continuously differentiable for $0 \leq t<h$. Since $y_{1}(t)$ is differentiable for $-\eta h \leq t<0$, it suffices to establish the following two facts to conclude that $y_{1}(t)$ is differentiable also for $-\eta h \leq t<h$ :

(i) $y_{1}(t)$ is continuous at $t=0$.

(ii) The left and right derivatives of $y_{1}(t)$ exist at $t=0$, and they coincide with each other.

Since $\phi(t)$ is continuous, it follows from (A1) that the condition (i) is equivalent to the condition $y_{1}(0)=\phi(0)$. We see that this is indeed satisfied if we note from (9) and $(22)$ that $y_{1}(0)=$ $q(0)+\sum_{i=1}^{\eta} D_{i} \phi(-i h)$; since $D_{i}=K_{i}$ by (18), it follows from (19) and (21) that $y_{1}(0)=\xi=\phi(0)$.

We next consider the condition (ii). Since $y_{1}(t)=\phi(t),-\eta h \leq t \leq 0$ under (i), it follows from the continuous differentiability of $\phi(t)$ over $-\eta h \leq t \leq 0$ that the left-derivative of $y_{1}(t)$ exists at $t=0$ and coincides with that of $\phi(t)$ at $=0$, denoted by $\dot{\phi}(-0)$ :

$$
\dot{y}_{1}(-0)=\dot{\phi}(-0)
$$

On the other hand, by $(16)^{1}$, the right-derivative of $y_{1}(t)$ at $t=0$ is given by

$$
\dot{y}_{1}(+0)=\dot{q}(+0)+\sum_{i=1}^{\eta} D_{i} \dot{y}_{1}(-i h)
$$

Hence, the condition (ii) can be rewritten as

$$
\dot{q}(+0)=\dot{\phi}(-0)-\sum_{i=1}^{\eta} D_{i} \dot{\phi}(-i h)
$$

To eliminate $q$ from this condition, we introduce a different expression for the left hand side; it can be represented as

$$
\dot{q}(+0)=A q(0)+\sum_{i=1}^{\eta} B_{i} \phi(-i h)
$$

from (8) and (22). It follows from (18), (19) and (21) that

$$
q(0)=\phi(0)-\sum_{i=1}^{\eta} D_{i} \phi(-i h)
$$

\footnotetext{
${ }^{1}$ Recall that the present proof aims at validating, for $t \geq 0$, the differentiation of (15) to arrive at (16). Hence, it is obviously inappropriate to use (16) directly here. Nonetheless, however, it has been established in the above that (15) is validated for $0 \leq t<h$, and the left hand side and the second term on the right hand side are continuously differentiable for $0 \leq t<h$. Hence, the resulting (A4) can be validated by taking the right-derivative at $t=0$.
} 
and substituting it into (A6), we have

$$
\dot{q}(+0)=A \phi(0)+\sum_{i=1}^{\eta}\left(B_{i}-A D_{i}\right) \phi(-i h)
$$

The condition (A5) can be rewritten by replacing its left hand side with the right hand side of (A8), but this rearranged condition indeed holds by (20) because of (18).

Summarizing the above arguments, we have established the former half of the proof as desired: if $\phi(t)$ that is continuously differentiable for $-\eta h \leq t \leq 0$ satisfies the condition (20) (i.e., if $y_{1}(t)=\phi(t),-\eta h \leq t \leq 0$ satisfies the DDE (1) at $\left.t=0\right)$ then $y_{1}(t)$ resulting from $\Sigma^{\mathrm{I}}$ under the initial conditions (21) and (22) is (continuous and) continuously differentiable for $-\eta h \leq t<h$ (and thus in particular for $-(\eta-1) h \leq t<h)$. Hence by the arguments of Section 4.1, this $y_{1}(t)$ satisfies $(1)$ over $-(\eta-1) h \leq t<h$. The last consequence implies that

(a) The condition corresponding to (20) with the time advanced by $h$ also holds.

(To validate rigorously this argument about the time advance by $h$, we must notice and then resolve the problem that $y_{1}(t)$ in the foregoing arguments is defined only on $-\eta h \leq t<h$ and thus $y_{1}(h)$ has not been defined; this leads to a further obstacle in defining the left-derivative of $y_{1}(t)$ at $t=h$. However, $\lim _{t \rightarrow h-0} y_{1}(t)$ does exist and thus $y_{1}(h)$ can be defined through continuity; then the left-derivative $\dot{y}_{1}(h-0)$ also becomes well-defined, which in fact coincides with $\lim _{t \rightarrow h-0} \dot{y}_{1}(t)$.) On the other hand,

(b) The condition corresponding to (19) with the time advanced by $h$ holds automatically provided that $x(h)$ is defined through continuity ${ }^{1}$ as required by the property of a regular solution by definition.

In addition, we see from (9), (10) and (A2) that the relation corresponding to (A2) holds with the time advanced by $h$, i.e.,

(c) $u_{i}(t)=y_{1}(t-i h), h \leq t<2 h, i=1, \cdots, \eta$ holds; this implies that the input of $F^{\mathrm{I}}$ over $h \leq t<2 h$ is given by the relation corresponding to (22) with the time advanced by $h$,

and also implies $u_{i}(h)=y_{1}(-(i-1) h)=\phi(-(i-1) h), i=1, \cdots, \eta$. Hence, (9) evaluated at $t=h$, i.e.,

$$
q(h)=y_{1}(h)-\sum_{i=1}^{\eta} D_{i} u_{i}(h)
$$

can be rewritten as

$$
q(h)=x(h)-\sum_{i=1}^{\eta} K_{i} \phi(-(i-1) h)
$$

where we used $y_{1}(h)=x(h)$ by continuity. This implies that

(d) The state of $F^{\mathrm{I}}$ at $t=h$ satisfies the condition corresponding to (21) with the time advanced by $h$.

From the facts (a)-(d) shown above regarding the time advance by $h$, we can repeat the arguments by regarding $t=h$ as the initial time instant; this immediately leads to that $y_{1}(t)$ is (continuous and) continuously differentiable and coincides with the regular solution $x(t)$ of the DDE (1) over $t \geq-\eta h$. This completes the proof.

Q.E.D. ${ }^{1}$ Since $y_{1}(t)=x(t)$ is continuous for $-\eta h \leq t<h$, the existence of $\lim _{t \rightarrow h-0} y_{1}(t)$ mentioned above ensures that of
$\lim _{t \rightarrow h-0} x(t)$. That is, we define $x(h)=y_{1}(h)$. 
Proof of Theorem 2. The proof is based on the modified form (6) of the neutral DDE (1).

We first note that solving the second equation of the modified DDE (6) for $x(t)$ and substituting it into the first equation of (6) lead to

$$
\frac{d}{d t} v(t)=J v(t)+\sum_{i=1}^{\eta} B_{i} x(t-i h)
$$

where we have used $L_{i}+J K_{i}=B_{i}$. For $0 \leq t<h,(\mathrm{~A} 11)$ can be rewritten as

$$
\frac{d}{d t} v(t)=J v(t)+\sum_{i=1}^{\eta} B_{i} \phi(t-i h)
$$

and comparing this with (8) leads to $v(t)=q(t)(0 \leq t<h)$ if we note (22); this is because $v(0)=q(0)$ by $(2),(3)$ and (21), and also $A=J$. This in particular implies that $v(t)$ (and thus also $x(t))$ is continuously differentiable for $0 \leq t<h$ by the assumption on $\phi(t)$.

Regarding $\Sigma^{\mathrm{I}}$, on the other hand, it follows by applying similar arguments to those at the beginning of the proof of Theorem 1 that (15) holds for $0 \leq t<h$ under the initial input (22). Since $x(t-i h)=y_{1}(t-i h)(i=1, \cdots, \eta)$ for $0 \leq t<h$, it follows by the comparison of (15) with the second equation of $(6)$ that $v(t)=q(t)(0 \leq t<h)$ obviously implies $y_{1}(t)=x(t)(0 \leq t<h)$.

Also, as long as we consider continuous $q(t)$ for $\Sigma^{\mathrm{I}}$ and continuous $v(t)$ for the DDE (6) (as is indeed the case with the definition of a pseudo concatenated solution and this theorem), $v(t)=q(t)(0 \leq t<h)$ implies $^{1} v(h)=q(h)$, and hence we have ${ }^{2}$

$$
\begin{aligned}
q(h) & =v(h) \\
& =x(h)-\sum_{i=1}^{\eta} K_{i} x(-(i-1) h) \\
& =x(h)-K_{1} \xi-\sum_{i=2}^{\eta} K_{i} \phi(-(i-1) h)
\end{aligned}
$$

This implies that the "initial value" of $q$ that we view by advancing the time by $h$ is given by the relation corresponding to (21) with the time advanced by $h$. In addition, we can readily see from (9), (10) and (A2) that the "initial input" (under the time advance by $h$ ) of $F^{\mathrm{I}}$ for $h \leq t<2 h$ is given by the relation corresponding to (22) with the time advanced by $h$. These two facts about the time advance by $h$ enable us to repeat the arguments, which immediately leads to the completion of the proof.

Q.E.D.

Proof of Theorem 3. We first note that $y_{1}(t)=q(t), t \geq 0$ from (9) since $D_{i}=0, i=1, \cdots, \eta$ by (18). Hence, when we define $y_{1}(t),-\eta h \leq t<0$ by (A1), it follows from (8) and (22) that

$$
\dot{y}_{1}(t)=A y_{1}(t)+\sum_{i=1}^{\eta} B_{i} y_{1}(t-i h)
$$

for $0 \leq t<h$. Since $y_{1}(0)=q(0)=\xi$ by the hypothesis, $y_{1}(t)=q(t)$ resulting from $\Sigma^{\mathrm{I}}$ coincides with the regular solution $x(t)=: x^{0}(t)$ of $(7)$ for $0 \leq t<h$. This $y_{1}(t)$ is continuous at $t=0$, while $y_{1}(t)=\phi(t)$ is also continuous at $t=-i h(i=1, \cdots, \eta-1)$. Hence, if we note (10), we see from (8) that $q(t)\left(=y_{1}(t)\right)$ resulting from $\Sigma^{\mathrm{I}}$ is differentiable at $t=h$. Since this in particular implies

\footnotetext{
${ }^{1}$ The boundedness assumption on $\phi(t)$ ensures the existence of the limit for $t \rightarrow h-0$ as to the solution $q(t)$ of the differential equation (8), and $v(h)$ is defined as the value of the limit.

${ }^{2}$ (A13) can be regarded as the equation that defines the value of $x(h)$ for the pseudo concatenated solution of the DDE (6), from the value that has already been determined for $v(h)$.
} 
that $y_{1}(t)$ is continuous at $t=h$, we have (i) $q(h)=y_{1}(h)=\lim _{t \rightarrow h-0} y_{1}(t)=\lim _{t \rightarrow h-0} x(t)$. Also, it is easy to see that (ii) the input of $\Sigma^{\mathrm{I}}$ for $h \leq t<2 h$ is given by

$$
u_{i}(t)=\left\{\begin{array}{l}
y_{1}(t-h)(i=1) \\
\phi(t-i h)(i=2, \cdots, \eta)
\end{array}\right.
$$

which corresponds to the input given by (22) with the time advanced by $h$. It follows from (i) and (ii) that we can repeat the above arguments by advancing the time by $h$, and we can conclude that $y_{1}(t)=q(t)$ resulting from $\Sigma^{\mathrm{I}}$ for $h \leq t<2 h$ coincides with the regular solution $x(t)=: x^{1}(t)$ of $(7)$ with $t=h$ and $x(h):=\lim _{t \rightarrow h-0} x(t)$ viewed as the initial time and initial state, respectively. Recalling that $y_{1}(t)$ is differentiable at $t=h$, we see that the function obtained by concatenating $x^{0}(t), 0 \leq t<h$ and $x^{1}(t), h \leq t<2 h$ is (continuous and) differentiable at $t=h$. This implies that $x^{1}(t)$ is indeed the regular solution of (7) with $t=0$ viewed as the initial time, or equivalently, $q(t)$ is the regular solution of (7) for $0 \leq t<2 h$. Repeating these arguments leads to the assertion of the theorem.

Q.E.D.

Proof of Theorem 4. In the proof of Theorem 3, the arguments for showing the differentiability of $x(t)$ at $t=k h$ become irrelevant. Hence, the proof proceeds with a weaker hypothesis on $\phi(t)$ as long as the existence of $\lim _{t \rightarrow h-0} q(t)$ can be ensured. This is indeed the case under the hypothesis of the theorem, and thus the assertion of the theorem follows.

Q.E.D. 